\title{
TO EVALUATE THE EFFICACY OF AMLAKYADI CHURNAM IN MANAGEMENT OF GARBHINI PANDU
}

\author{
*D Anuradha ${ }^{1}$, N Anish ${ }^{2}$, NMA Rasheed ${ }^{3}$, M Srinivasulu. ${ }^{4}$ \\ ${ }^{1 \& 4}$ Dr. B.R.K.R. Govt. Ayurvedic College, opp.E.S.I. Hospital, A.G. Colony Road, Erragaddda Hyderabad-500038, \\ ${ }^{2}$ AIZANT Drug Research Solutions, Dhulapalli, Hyderabad, India \\ ${ }^{3}$ Central Research Institute of Unani Medicine, opp.E.S.I. Hospital, A.G. Colony Road, Erragaddda, Hyderabad-500038, India \\ *Corresponding Author's Detail: Asst.Prof. and HOD.Agadathanthra and vyavahara ayurveda Dr.BRKR.government ayurvedic medical \\ college, Hyderabad, Andhra Pradesh.Email:dranud@yahoo.com
}

\begin{abstract}
Female is the centre of human life circle. Ancient ethics like Manusmriti has evaluated female by giving prior position in human life. Prajadhari (giving birth) is the most important role. For those women needs healthy conditions during pregnancy. In this physiological process mother experiences certain problems like pandu (Anemia).If it is neglected it may lead to complicate events of pregnancy, labor \& purperum etc. there is marked demand of extra iron during pregnancy especially in the second half. Even an adequate diet cannot provide the extra demand of iron. The fall in the hemoglobin concentration during pregnancy is due to combined effect of haemodilution and negative iron balance. The anemia is normocytic and normochromic type. pandu by amlakyadi choornam consists of Amlaki, Yast \& Swarna maukshika. Amalaki is Panchersa (devoid of lavana) and pitta samake. Poorva malabedhanakari (laxative). It consists of vit-C which helps in good obserption of loya yast is Madhurarasa rasayana, balya, ruchikara Sw.M.Entaim loha \& tamtra. Known for rasaguna, raktaprasadake, rasantpadaka, pitta shameke etc. tamra has got krimighna property Mudhu:- Yogavali, Srothoshodana, Antiemitic. The pregnant woman should be treated with the use of soft, sweet, cold, pleasing \& gentle dugs, dietetics and behavior. Present clinical study is on grabhinipandu(anemia in pregnancy) with Amlakyadi choornam which contains Amlaki, yatimadhu and swarnamakshikabhasma. Pregnant women were selected for clinical trial at O.P.D. of GAH Erragadda. 3grms of Amlakyadi churnam was given with anupanum of madhu (honey) twice daily after light food in the morning \& evening for 90 days. This compound drug is of selective rasayanas. It is economical, safe \& effective and devoid complications. Total 30 patients were treated, parameters are assessed monthly once throughout the period of 90 days. At the same time clinical symptoms like anorexia, poor appetite, Shotha (inflammation), malabandhata (constipation), Pallor etc and $\mathrm{Hb} \%$ also observed. Weight gain observed in all the patients and also $\mathrm{Hb} \%$ is improved in all the cases. There were no side effects noticed while usage well tolerated by the pregnant woman without complications.
\end{abstract}

Key Words: Pandu roga, Garbhini,Amlakyadi churna and women.

\section{INTRODUCTION:}

Pandu (anaemia) is one of the diseases of rasavaha srotodhusti, Pregnancy is a physiological process, during this period mother experiences certain problems like Pandu. $_{1}$ Negligence of these problems may lead to complicate events of pregnancy, labor and purperum.Garbhini should be treated just like a pot filled with oil, slightest oscillation of such pot causes spilling of oil. Similarly slightest excitement to the pregnant woman can initiate abortion, etc. ${ }^{2}$

Pregnancy makes the women more susceptible to develop Pandu(anaemia) not only because of extra demand of the growing fetus but also due to the deficient diet. Incidence of disease ranges from $40-80 \%$. It is a universal problem and occurs for any age group of persons.

Pregnancy is a state of continuous physical adaptation to meet and anticipate the demands of the growing fetus and to provide a stable environment in which it growth can take place for fetal needs ${ }^{3}$

Hydroemia of pregnancy (fall in hemoglobin level) is due to increase in red cell volume is less than $20 \%$ though the plasma volume will increase by $40 \% 11.0 \mathrm{gms} / 100 \mathrm{ml}$ of peripheral blood @ less than that is known to be anemia. ${ }^{4}$

\section{MATERIALS \&METHODS:}

\section{DRUGS}

1. Amlaki(phyllanthusemblicalinn)2.Yastimadhu(Gl ycyrrhizgobra)3.Swarnamakeshika(Copper pyrite $\left.\mathrm{cuFes}_{2}\right) .^{5}$

Anupana- Madhu.

Preparation of drug: - Fruits of Amlaki \& roots of Yastimadhu were prepared as fine powder. Swarnamakshka bhasma prepared according to process mentioned in Rasataranghi book.

yasti + amalki \& Swarnabhasma were taken in the ratio of 3:1. 3gms. of this drug (Amalakyadichurn) was given with Madhu twice daily (orally) after light food intake in the morning \& evening for 90 days continuously. ${ }^{6}$

Patients: 30 patients randomly selected who were suffering from Garbhini Pandu from the Department of Prasuti at G.A.H- Erragadda,Hyderabad.

\section{Parameters:}

1) Objective: - $\mathrm{Hb} \%$ \& weight

2) Subjective: - Anorexia, nausea, vomiting, heartburn, constipation, shotha, pallor etc

\section{Follow up:}

1) Assessment after every month

2) Signs \& Symptoms recorded before \& after treatment. 
Table 1: Based on Age

\begin{tabular}{|c|c|c|c|}
\hline Sl.No & Age & No. of Cases & Percentage \\
\hline 1 & $18 Y r s-22$ Yrs & 15 & $50 \%$ \\
\hline 2 & 23 Yrs - 27Yrs & 9 & $30 \%$ \\
\hline 3 & $28 Y r s-32$ Yrs & 6 & $20 \%$ \\
\hline & & & \\
\hline
\end{tabular}

Table 2 : Based on Occupation

\begin{tabular}{|c|c|c|c|}
\hline S.No. & Occupation & No. of Cases & Percentage \\
\hline 1 & House wife & 15 & 50 \\
\hline 2 & Employee & 9 & 30 \\
\hline 3 & Others (labor) & 6 & 20 \\
\hline
\end{tabular}

Table 3: Based on Socio-Economic status

\begin{tabular}{|c|c|c|c|}
\hline S.No. & S.E.Status & No. of Cases & Percentage \\
\hline 1 & Low & 12 & 40 \\
\hline 2 & Middle & 9 & 30 \\
\hline 3 & Upper & 9 & 30 \\
\hline
\end{tabular}

Table 4: Based on Dietary habits

\begin{tabular}{|c|c|c|c|}
\hline S.No. & Dietary Habits & No. of Patients & Percentage \\
\hline 1 & Vegetarian & 21 & 70 \\
\hline 2 & Mixed diet & 9 & 30 \\
\hline
\end{tabular}

Table 5: Based on Gravid

\begin{tabular}{|c|c|c|c|}
\hline Sl.No. & Gravida & No. of Cases & Percentage \\
\hline 1 & Primary Gravida & 8 & 26.66 \\
\hline 2 & Secondary Gravida & 14 & 46.68 \\
\hline 3 & Multi Gravida & 8 & 26.66 \\
\hline
\end{tabular}

Table 6: Based on $2^{\text {nd }}$ Trimestar

\begin{tabular}{|c|c|c|c|}
\hline Sl.No. & $\mathbf{2}^{\text {nd }}$ Trimester & No. of Cases & Percentage \\
\hline 1 & $4^{\text {th }}$ month & 9 & 30.0 \\
\hline 2 & $5^{\text {th }}$ month & 11 & 36.67 \\
\hline 3 & $6^{\text {th }}$ month & 10 & 33.33 \\
\hline
\end{tabular}

Table 7: Based on $\mathrm{Hb} \%$

\begin{tabular}{|c|c|c|c|}
\hline Sl.No. & HB\% & No. of Cases & Percentage \\
\hline 1 & $7.0 \mathrm{gm}-8.0 \mathrm{gm}$ & 8 & 26.66 \\
\hline 2 & $8.0 \mathrm{gm}-9.0 \mathrm{gm}$ & 14 & 46.88 \\
\hline 3 & $9.0 \mathrm{gm}-10.0 \mathrm{gm}$ & 8 & 26.66 \\
\hline
\end{tabular}

Table 8: Based on Signs \& Symptoms

\begin{tabular}{|c|c|c|c|}
\hline Sl.No. & Signs \& Symptoms & No. of Cases & Percentage \\
\hline 1 & Anorexia & 15 & 50 \\
\hline 2 & Poor appetite & 21 & 70 \\
\hline 3 & Heart burn & 18 & 60 \\
\hline 4 & Constipation & 15 & 50 \\
\hline 5 & Shotha(edema) & 9 & 30 \\
\hline 6 & General weakness & 18 & 60 \\
\hline 7 & Pallor (conjunctiva, tongue\& nails) & 12 & 73.33 \\
\hline
\end{tabular}


Table 9: results based on Age

\begin{tabular}{|c|c|c|c|c|}
\hline Age & No. of Cases & Relief Completely & Relief partial & No relief \\
\hline 18Yrs -22Yrs & 15 & $66.66 \%(10)$ & $26.66 \%(4)$ & $6.66 \%(1)$ \\
\hline 23Yrs-27Yrs & 9 & $77.77 \%(7)$ & $11.11 \%(1)$ & $11.11 \%(1)$ \\
\hline 28 Yrs-32Yrs & 6 & $66.66 \%(4)$ & $16.66 \%(1)$ & $16.66 \%(1)$ \\
\hline
\end{tabular}

Table 10: results based on Occupation

\begin{tabular}{|c|c|c|c|c|}
\hline Occupation & No. of Cases & Relief Completely & Relief partial & No relief \\
\hline House wife & $\mathbf{1 5}$ & $66.66 \%(10)$ & $26.66 \%(4)$ & $6.66 \%(1)$ \\
\hline Employee & $\mathbf{9}$ & $77.77 \%(7)$ & $11.11 \%(1)$ & $11.11 \%(1)$ \\
\hline Others (labor) & $\mathbf{6}$ & $66.66 \%(4)$ & $16.66 \%(1)$ & $16.66 \%(1)$ \\
\hline
\end{tabular}

Table 11: results based on Socio - Economic status

\begin{tabular}{|c|c|c|c|c|}
\hline S.E.S & No. of Cases & Relief Completely & Relief partial & No relief \\
\hline Low & $\mathbf{1 2}$ & $50 \%(6)$ & $33.33 \%(4)$ & $16.66 \%(2)$ \\
\hline Middle & $\mathbf{9}$ & $66.66 \%(6)$ & $22.22 \%(2)$ & $11.11 \%(1)$ \\
\hline Upper & $\mathbf{9}$ & $100 \%(9)$ & - & - \\
\hline
\end{tabular}

Table 12: Result based on Diet habits

\begin{tabular}{|c|c|c|c|c|}
\hline Dietary Habits & No. of Cases & Relief Completely & Relief partial & No relief \\
\hline Vegetarian & $\mathbf{2 1}$ & $55.55 \%(6)$ & $33.33 \%(3)$ & $11.11 \%(1)$ \\
\hline Mixed diet & $\mathbf{9}$ & $76.19 \%(16)$ & $14.28 \%(3)$ & $9.5 \%(2)$ \\
\hline
\end{tabular}

Table 13: results based on Gravida

\begin{tabular}{|c|c|c|c|c|}
\hline Gravida & No. of Cases & Relief Completely & Relief partial & No relief \\
\hline Primary Gravida & $\mathbf{8}$ & $62.5 \%(5)$ & $25.00 \%(2)$ & $12.50 \%(1)$ \\
\hline Secondary Gravida & $\mathbf{1 4}$ & $78.57 \%(11)$ & $14.44 \%(2)$ & $7.14 \%(1)$ \\
\hline Multi Gravida & $\mathbf{8}$ & $62.05 \%(5)$ & $25 \%(2)$ & $12.05(1)$ \\
\hline
\end{tabular}

Table 14: results based on $2^{\text {nd }}$ Trimestar

\begin{tabular}{|c|c|c|c|c|}
\hline $\mathbf{2}^{\text {nd }}$ Trimester & No. of Cases & Relief Completely & Relief partial & No relief \\
\hline $\mathbf{4}^{\text {th }}$ month & $\mathbf{9}$ & $66.66 \%(6)$ & $22.22 \%(2)$ & $11.11 \%(1)$ \\
\hline $\mathbf{5}^{\text {th }}$ month & $\mathbf{1 1}$ & $72.72 \%(8)$ & $18.18 \%(2)$ & $9.09 \%(1)$ \\
\hline $\mathbf{6}^{\text {th }}$ month & $\mathbf{1 0}$ & $70 \%(7)$ & $20 \%(2)$ & $10(1)$ \\
\hline
\end{tabular}

Table 15: results based on $\mathrm{Hb} \%$ before $\&$ after treatment

\begin{tabular}{|c|c|c|c|}
\hline Hemoglobin HB\% & No. of Cases & Before treatment & After treatment \\
\hline $7.0 \mathrm{gm}-\mathbf{8 . 0 g m}$ & 8 & $6.8 \mathrm{gms} \%$ & $11.05 \mathrm{gms} \%$ \\
\hline $\mathbf{8 . 0 g m}-9.0 \mathrm{gm}$ & 14 & $8.43 \mathrm{gms} \%$ & $12.09 \mathrm{gms} \%$ \\
\hline $\mathbf{9 . 0 g m}-10.0 \mathrm{gm}$ & 8 & $9.2 \mathrm{gms} \%$ & $12.91 \mathrm{gms} \%$ \\
\hline
\end{tabular}

Table 16: based on Signs \& Symptoms

\begin{tabular}{|c|c|c|c|c|}
\hline \multirow{2}{*}{ Signs \& Symptoms } & \multirow{2}{*}{ Before treatment } & \multicolumn{3}{|c|}{ After Treatment } \\
\hline & & Complete & Partial & No results \\
\hline Anorexia & $50 \%(15)$ & $73.33 \%(11)$ & $20 \%(3)$ & $6.66 \%(1)$ \\
\hline Poor appetite & $70 \%(21)$ & $61.90 \%(13)$ & $28.57 \%(6)$ & $9.52 \%(2)$ \\
\hline Heart burn & $60 \%(18)$ & $61.11 \%(12)$ & $27.77 \%(4)$ & $11.11 \%(2)$ \\
\hline Constipation & $50 \%(15)$ & $60 \%(9)$ & $26.66 \%(4)$ & $13.33 \%(2)$ \\
\hline Shotha & 30\% (9) & $55.55 \%(5)$ & $22.22 \%(2)$ & $22.22 \%(2)$ \\
\hline General weakness & $60 \%(18)$ & 83.33\% (15) & $11.11 \%(2)$ & $5.55 \%(1)$ \\
\hline Pallor (conjunctiva, tongue nails) & 73.33\% (22) & $81.81 \%(18)$ & $9.09 \%(2)$ & $9.09 \%(2)$ \\
\hline
\end{tabular}


Table 17: total results of 30 patients

\begin{tabular}{|c|c|c|c|}
\hline No. of cases & Complete relief & Partial relief & No relief \\
\hline 30 & $70.34 \%$ & $19.49 \%$ & $10.16 \%$ \\
\hline
\end{tabular}

\section{DISCUSSION:}

The pregnant women requires additional amount of energy and nutrition as she has to supply the fetus which may lead to nutritional deficiency disorders like Pandu, etc. the present study is aimed at finding as safe effective, management and to get Garbhini into normal from Pandu by Amalakyadi churna. Vitamin $-\mathrm{C}$ which is present in Amalaki will enhance the absorption of loha (in swarna makshika). In total the drug is tridoshashamaka, rasayana (health tonic), balya (energy), ruchikara. ${ }^{7 \& 8}$ (taste), jeavaneya, and brimhana(improves blood\&gives energy) in nature.

\section{REFERENCES:}

1. Agnivesa - Charka Samhita revised by charka \& Dhridabaka $5^{\text {th }}$ edition - Chowkamba Sanskrit Samsthana Varanasi-2001Ch.Ch.16, Ch.S.27/9-10.

2. A.L.Mudaliar - Clinical obstetrics - Novena offset printing co, Chennai $-9^{\text {th }}$ edition -1990 .

3. Text book of obstetrics - D.C.Dutte - Sree mote arati dry ref Yogaratnakaraswmabneera Dawn CIT Road, Calcutta- $10^{\text {th }}$ edition.

4. P.V.Tewari - Ayurveda Prasuthtantra evam stree roga part-1Chowkhamba Sanskrit series Varanasi - 1- 2003.

5. K.Nadkarni - Indian Materia Media - Popular Prakesham Pvt.Ltd, Bombay $3^{\text {rd }}$ edition -1975 .
The anupana madhu also having iron content, yogavahi in nature, with this nature of drug in all 30pts. weight gain observed and also $\mathrm{Hb} \%$ is improved.

\section{CONCLUSION:}

The compound preparation of Amalakyadi churnam contains Amalaki, Yasti \& Swarnamakshika. Madhu as anupana is very much useful in the management of Garbhini pandu. ${ }^{9}$ The drug is well tolerated by the pregnant women without any complications.

6. Dalhana - Susrutha Samhita - Nirnaya Sagar press Bombay 1974 - Su.Su.35/12

7. Charaka samhita with chakrapani commentary by Ganga sahey pande choukamba publication (1980).

8. Chistopher .J.Tibbs \& Heather M.Smith, Clinicians guide to viral hepatitis, Arnold publis lcatiohs London (2001).Ch.Ch.4/6/16, Ch.Ch-4/23, 7-11/16. Cu.u14/Sharangadhara- 1/34/4

9. Bhavaprakasha of Bhavamisra with English translation Translated by Prof.K.R.Srikantha Murthy, Krishnadas Academy, Varanasi $1^{\text {st }}$ edition 1998. 